\title{
Biomarkers of Unfavorable Outcome in Acute Ischemic Stroke Patients with Successful Recanalization by Endovascular Thrombectomy
}

\author{
Nailiang Zang Zhenzhou Lin Kaibin Huang Yue Pan Yanan Wu \\ Yongming Wu Shengnan Wang Dongmei Wang Zhong Ji Suyue Pan \\ Department of Neurology, Nanfang Hospital, Southern Medical University, Guangzhou, China
}

\section{Keywords}

Acute ischemic stroke - Endovascular thrombectomy .

Futile recanalization · Early complication · Biomarkers

\begin{abstract}
Background: We aimed to identify plasma markers of unfavorable outcomes for patients with acute ischemic stroke (AIS) after recanalization by endovascular thrombectomy (EVT). Methods: From November 2017 to May 2019, we prospectively collected 61 AIS patients due to anterior large vessel occlusion who achieved recanalization by EVT. Plasma samples were obtained between 18 and $24 \mathrm{~h}$ after recanalization. Unfavorable outcomes included futile recanalization at 90 days and overall early complications within 7 days after EVT. Results: After adjustment for age and initial National Institute of Health Stroke Scale (NIHSS), matrix metalloproteinase-9 (MMP-9), tenascin-C, thioredoxin, ADAMTS13, and gelsolin were independently associated with both futile recanalization and overall early complications significantly (all $p<0.05$ ), while $C$-reactive protein (CRP) was independently associated with overall early complications ( $p=0.031)$ but at the limit of significance for futile recanalization $(p=0.051)$. The baseline clinical model (BCM) (including age and initial
\end{abstract}

karger@karger.con www.karger.com/ced

(c) 2020 S. Karger AG, Base

Karger'
NIHSS) demonstrated discriminating ability to indicate futile recanalization (area under the curve [AUC] $0.807,95 \%$ confidence interval $[\mathrm{Cl}]$ 0.693-0.921) and overall early complications (AUC 0.749, 95\% Cl 0.611-0.887). BCM+MMP-9+ thioredoxin enhanced discrimination (AUC $0.908,95 \% \mathrm{Cl}$ $0.839-0.978, p=0.043$ ) and reclassification (net reclassification improvement [NRI] $67.2 \%, p<0.001$ ) to indicate futile recanalization. With respect to overall early complications, $\mathrm{BCM}+\mathrm{MMP}-9+$ tenascin-C, BCM+MMP-9+CRP, BCM+MMP9+ADAMTS13,BCM+tenascin-C+ADAMTS13, and BCM+CRP+ ADAMTS13, all improved discrimination (AUC [95\% Cl]: 0.868 $[0.766-0.970], 0.882[0.773-0.990], 0.886$ [0.788-0.984], 0.880 [0.783-0.977], and 0.863 [0.764-0.962], respectively, all $p<0.05$ by the DeLong method) and reclassification (NRI $59.1 \%, 71.8 \%, 51.1 \%, 67.4 \%$, and $38.3 \%$, respectively, all $p<$ 0.05). Conclusions: The increased levels of MMP-9, tenascinC, CRP, thioredoxin, and decreased levels of ADAMTS13 and gelsolin were independent predictors of futile recanalization in AIS patients after recanalization by EVT.

(c) 2020 S. Karger AG, Basel

Nailiang Zang and Zhenzhou Lin contributed equally to this study.

Suyue Pan or Zhong Ji

Department of Neurology, Nanfang Hospital, Southern Medical University

1838 North Guangzhou Avenue

Guangzhou 510515 (China)

pansuyue@smu.edu.cn or jizhong@ fimmu.com 


\section{Introduction}

Endovascular thrombectomy (EVT) is a safe and effective treatment for acute ischemic stroke (AIS) caused by intracranial large vessel occlusion (LVO) in anterior circulation [1]. Despite successful recanalization via EVT, some patients develop early complications of AIS during the acute phase, including early neurological deterioration (END) [2], symptomatic hemorrhagic transformation (sHT) [3], brain herniation [4], and malignant brain edema (MBE) [5]. Besides, almost half of the patients remain functional dependence (modified Rankin Scale [mRS], 3-6) at 3 months after onset, which is thus called futile recanalization [6-8]. Prompt identification of patients at increased risks of unfavorable outcomes may help target patients who deserve close attention and timely treatments.

In previous studies, higher initial National Institute of Health Stroke Scale (NIHSS) score, older age, and longer time from onset to treatment were associated with poor AIS outcomes after EVT $[6,8,9]$. Aside from clinical parameters, blood biomarkers may also serve as a practical tool to represent the pathophysiology status before clinical deterioration. A number of studies have identified that various blood biomarkers, such as matrix metalloproteinase-9 (MMP-9) and C-reactive protein (CRP), are associated with functional outcomes of AIS $[10,11]$. However, few studies have addressed the relationship of blood biomarkers with unfavorable outcomes in AIS patients who achieve successful recanalization via EVT. Therefore, we prospectively collected AIS patients with successful recanalization by EVT and investigated the prognostic plasma biomarkers that have potential association with the pathophysiology of AIS.

\section{Methods}

\section{Study Design and Patient Selection}

This was a single-center observational study undertaken at the NanFang Hospital between November 2017 and May 2019. Patients with AIS due to anterior circulation LVO who achieved successful recanalization by EVT using stent retrievers (SolitaireTM AB: ev3, Irvine, CA, USA) with local anesthesia were screened for eligibility of the study. Successful recanalization was defined as modified Thrombolysis in Cerebral Infarction (mTICI) grade, 2b-3 [12]. Patients received bridging therapy with intravenous thrombolysis if they met the AHA/ASA criteria [13]. We received informed consent from all patients or their families, and the Medical Ethics Committee of NanFang Hospital approved the study protocol (NFEC-2019-189).

The inclusion criteria were as follows: (1) AIS patients who underwent EVT for anterior circulation LVO; (2) aged $\geq 18$ years; (3) initial NIHSS on admission $\geq 6$; (4) ASPECTS based on diffusion weighted imaging (DWI) $\geq 6$; (5) had anterior circulation LVO confirmed by digital subtraction angiography, and the occlusion sites included intracranial terminal internal carotid artery and middle cerebral artery (M1 or proximal M2 segments or both); (6) EVT was performed with groin puncture initiated $<6 \mathrm{~h}$ from stroke onset, and those initiated within 6-16 h from onset were also eligible if they met the criteria in DEFUSE 3 trial [14]; (7) $\mathrm{mTICI}<2 \mathrm{~b}$ before thrombectomy and mTICI $\geq 2 \mathrm{~b}$ after thrombectomy.

The exclusion criteria were as follows: (1) unfavorable outcomes occurred before sampling; (2) had significant pre-stroke disability (pre-stroke $\mathrm{mRS} \geq 2$ ); (3) had evidence of intracranial hemorrhage, subarachnoid hemorrhage, arteriovenous malformation, aneurysm, or intracranial tumor verified by CT/MRI on admission; (4) had preexisting neurological or psychiatric disease that would confound neurological evaluation; (5) had active or recent hemorrhage and history of trauma or surgery within 2 months before stroke onset; (6) had concurrent infection at the time of sample collection; (7) had rheumatoid immune diseases, severe liver/kidney diseases, hematopathy, or malignant tumors; (8) suspicious of infectious embolus or bacterial endocarditis; (9) had previous endovascular therapy; (10) baseline platelet count $<50,000 / \mu \mathrm{L} ;$ (11) pregnant/lactating women; (12) missing clinical/ imaging/follow-up data or information; and (13) blood samples showed poor quality.

\section{Clinical Information}

All the patients were admitted to the intensive care unit after EVT for close neurological monitoring. We recorded the patients' clinical characteristics and information (including age, sex, time from onset to recanalization, initial NIHSS, occlusion site, fasting blood glucose, number of passes to achieve recanalization) and the complications during hospitalization. Stroke subtypes were determined by Trial of Org 10,172 in Acute Stroke Treatment classification. NIHSS score was recorded daily by trained neurologists (Z.Z.L. and K.B.H.) from admission until discharge. ASPECTS were measured based on DWI. The mRS score was acquired at 90 days after EVT by a trained neurologist (Y.P.) blind to other study data. CT was performed at $24 \mathrm{~h}$ after EVT before the initiation of antithrombotic treatment. Additional brain imaging was performed when the patient's status got deteriorated. Patient's blood pressure (BP) levels using invasive arterial measurements were collected as 24 values of systolic BP (SBP) and diastolic BP recorded at regular 60-min intervals within the first $24 \mathrm{~h}$ after EVT. The mean SBP and maximal SBP values within the first $24 \mathrm{~h}$ after EVT were collected. The treatment of elevated BP and target BP levels depended on attending neurologist's decision and clinical status of patients.

\section{Outcome Definitions}

Both futile recanalization and overall early complications were regarded as unfavorable outcomes in the present study. The primary outcome of this study was futile recanalization, defined as successful recanalization (achieved mTICI $\geq 2 \mathrm{~b}$ after thrombectomy) plus functional dependence (mRS $\geq 3$ at 90 days) following EVT [6-8]. The secondary outcome was overall early complication, defined as the occurrence of any early complications within 7 days after EVT, including END [2, 15, 16], sHT [2, 3], brain herniation, and MBE [5]. The definitions of each early complication are as follows: (1) END: $\geq 2$-point increment in NIHSS compared 
to the best neurological status within 7 days after EVT; (2) sHT: neurological worsening ( $\geq 4$-point increment in NIHSS) plus any hemorrhagic transformation with mass effect within 7 days after EVT, either within initial infarction site or distant area, confirmed by non-contrast computed tomography; (3) brain herniation: clinical symptoms of brain herniation (including level of consciousness decreased, pupil dilation with failure to constrict in response to light, or/and vomiting) plus brain tissue shifting signs confirmed by non-contrast computed tomography or MRI within 7 days after EVT; (4) MBE: parenchymal hypodensity of $\geq 50 \%$ of MCA territory, local brain swelling signs, and midline shift $\geq 5 \mathrm{~mm}$ at septum pellucidum or pineal gland with basal cistern obliteration within 7 days after EVT.

Plasma Collection and Biomarker Analysis

Peripheral blood of each included patient was drawn between 18 and $24 \mathrm{~h}$ after EVT. After centrifugation $\left(3,000 \mathrm{~g}, 4^{\circ} \mathrm{C}\right)$ for 10 min, plasma was blindly coded and stored at $-80^{\circ} \mathrm{C}$ until assayed by liquid chromatography-mass spectroscopy/mass spectroscopy (LC-MS/MS) analysis and parallel reaction monitoring (PRM) verification [17] (Wininnovate Bio, Shenzhen, China), the details of which are given in online suppl. material (for all online suppl. material, see www.karger.com/doi/10.1159/000510804).

First, we carried out a systematic search of literature on stroke biomarkers in the MEDLINE, Embase, and Web of Science databases, and we made a list (online suppl. Table 1) of common blood biomarkers associated with AIS. However, some of the biomarkers in the list failed to be detected in this study with the approach of LC-MS/MS analysis and PRM which were performed by the company (Wininnovate Bio, Shenzhen, China). The possible reason was that there were no already established protocols for searching the unique peptides of these proteins (which was the key step in LC-MS/MS and PRM) in the company. A total of 35 kinds of proteins were able to be detected using LC-MS/MS and PRM in this study, and only the following 6 biomarkers were found to be related to unfavorable outcomes, including MMP-9 (a marker of blood-brain barrier [BBB] disruption $[18,19]$ ), tenascin-C (involved in neuronal apoptosis and BBB disruption [20, 21]), CRP (an inflammatory marker $[22,23]$ ), thioredoxin (an indicator of oxidative stress [24-26]), a disintegrin and metalloproteinase with a thrombospondin motif repeats 13 (ADAMTS13, related to microcirculatory disturbances $[7,27]$ ), and gelsolin (implicated in microcirculatory disturbances [28, 29]).

\section{Statistical Analysis}

Protein intensity was defined as the arithmetic mean of the peptide peak intensities attributed to the protein [17]. Data were presented as mean (standard deviation), median (interquartile range $[\mathrm{IQR}]$ ), and number (percentage), as appropriate. Student's $t$ tests or Mann-Whitney $U$ tests were applied for continuous data with or without normal distributions, respectively. Pearson's $\chi^{2}$ tests or Fisher's exact tests were conducted for categorical data as appropriate. Univariate and multivariate analysis were applied to find the clinical variables and plasma biomarkers associated with unfavorable outcomes. The levels of all the biomarkers were recorded as continuous variable, which were used in univariate and multivariate analysis. A baseline clinical model (BCM) was established using clinical variables that were independently associated with unfavorable outcomes in multivariate regression analysis, then individual or combined plasma biomarkers were added into

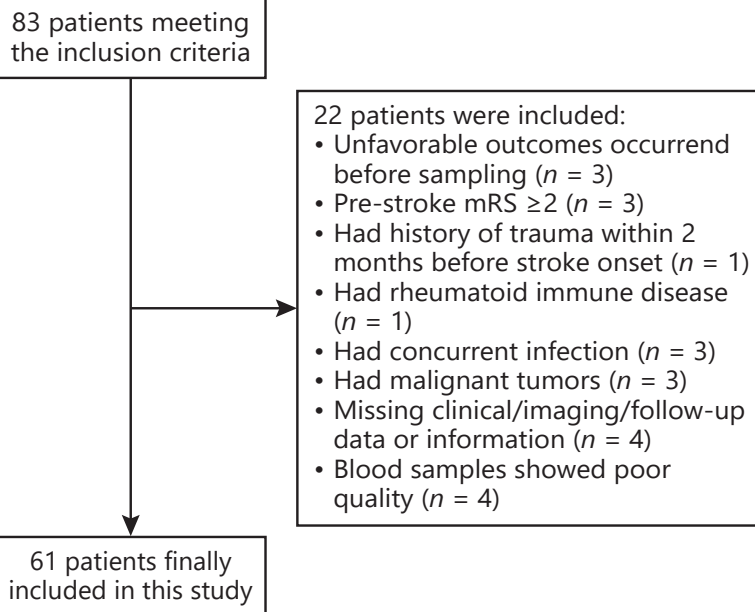

Fig. 1. Flowchart illustrating included and excluded patients in the present study. mRS, modified Rankin Scale.

BCM to investigate whether any of these biomarkers could improve the discriminating ability for unfavorable outcome. The ability to discriminate outcomes was assessed by receiver operating characteristic curve analysis and integrated discrimination improvement (IDI). The differences in area under the curve (AUC) between models were tested by the DeLong method. Besides, we calculated categorical net reclassification improvement (NRI) with the thresholds: $<0.20$ (low risk), $0.20-0.50$ (intermediate-low risk), $0.50-0.80$ (intermediate-high risk), and $>0.80$ (high risk) [30]. Statistical analysis was performed using SPSS (25.0), MedCalc (19.0.7), and $\mathrm{R}$ (3.5.2).

\section{Results}

\section{Clinical Characteristics}

The flowchart illustrating included and excluded patients in the present study is shown in Figure 1. In total, we prospectively selected 61 AIS patients with successful recanalization after EVT using SolitaireTM AB stent retrievers, among whom 34 patients had futile recanalization, and 21 patients developed overall early complications, involving 20 patients with END, 15 patients with MBE, 15 patients with brain herniation, and 9 patients with sHT. Among the 21 patients who developed overall early complications, 2 patients developed brain herniation at the time which was close to sampling time; thus, they were excluded for analysis in overall early complications, while they were available for analysis in futile recanalization. After recanalization, overall early complications started at a median time of 37 (IQR 27-52) h. The 
Table 1. Clinical characteristics of AIS patients who achieved successful recanalization by EVT between those with and without futile recanalization

\begin{tabular}{|c|c|c|c|}
\hline \multirow[t]{2}{*}{ Clinical characteristics } & \multicolumn{2}{|c|}{ Futile recanalization } & \multirow[t]{2}{*}{$p$ value } \\
\hline & yes $(n=34)$ & no $(n=27)$ & \\
\hline Age, median (IQR), years & $61(51-78)$ & $45(38-58)$ & $0.001^{\mathrm{e}}$ \\
\hline Initial NIHSS score, median (IQR) & $18(14-20)$ & $13(10-15)$ & $<0.001^{\mathrm{e}}$ \\
\hline Time from onset to recanalization, median (IQR), min & $322(285-441)$ & $385(247-484)$ & $0.727^{\mathrm{e}}$ \\
\hline Admission ASPECTS, median (IQR) & $7(6-9)$ & $8(7-9)$ & $0.308^{\mathrm{e}}$ \\
\hline Male, $n(\%)$ & $24(70.6)$ & $18(66.7)$ & $0.743^{\mathrm{f}}$ \\
\hline Treatment with intravenous thrombolysis, $n(\%)$ & $14(41.2)$ & $11(40.7)$ & $0.973^{\mathrm{f}}$ \\
\hline Number of passes $\geq 4$ times, $n(\%)$ & $5(14.7)$ & $1(3.7)$ & $0.214^{\mathrm{g}}$ \\
\hline Mean SBP, mean (SD), mm Hga & $131(8)$ & $125(16)$ & $0.067^{\mathrm{h}}$ \\
\hline Mean DBP, mean (SD), $\mathrm{mm} \mathrm{Hg}^{\mathrm{b}}$ & $74(8)$ & $73(11)$ & $0.848^{\mathrm{h}}$ \\
\hline SBP $\max \geq 160 \mathrm{~mm} \mathrm{Hg}, n(\%)^{\mathrm{c}}$ & $16(47.1)$ & $7(25.9)$ & $0.091^{\mathrm{f}}$ \\
\hline Fasting blood glucose, $\mathrm{mmol} / \mathrm{L}$ & $7.2(6.6-8.7)$ & $6.6(5.6-8.1)$ & $0.178^{\mathrm{e}}$ \\
\hline Site of occlusion & - & - & $0.255^{\mathrm{g}}$ \\
\hline tICA, $n(\%)$ & $6(17.6)$ & $5(18.5)$ & - \\
\hline MCA-M1 segment, $n(\%)$ & $27(79.4)$ & $18(66.7)$ & - \\
\hline MCA-M2 segment, $n(\%)$ & $1(2.9)$ & $4(14.8)$ & - \\
\hline Stroke subtype by TOAST criteria & - & - & $0.634^{\mathrm{g}}$ \\
\hline Large-artery atherosclerosis, $n(\%)$ & $13(38.2)$ & $11(40.7)$ & \\
\hline Cardioembolism, $n(\%)$ & $16(47.1)$ & $9(33.3)$ & \\
\hline Stroke of other determined etiology, $n(\%)$ & $2(5.9)$ & $3(11.1)$ & \\
\hline Stroke of undetermined etiology, $n(\%)$ & $3(8.8)$ & $4(14.8)$ & \\
\hline Hypertension, $n(\%)$ & $18(52.9)$ & $10(37.0)$ & $0.216^{\mathrm{f}}$ \\
\hline Diabetes mellitus, $n(\%)$ & $7(20.6)$ & $4(14.8)$ & $0.740^{\mathrm{g}}$ \\
\hline Hyperlipemia, $n$ (\%) & $6(17.6)$ & $5(18.5)$ & $0.930^{\mathrm{g}}$ \\
\hline Atrial fibrillation, $n(\%)$ & $12(35.3)$ & $6(22.2)$ & $0.266^{\mathrm{f}}$ \\
\hline Smoke, $n(\%)$ & $13(38.2)$ & $8(29.6)$ & $0.482^{\mathrm{f}}$ \\
\hline Congestive heart failure, $n(\%)$ & $4(11.8)$ & $1(3.7)$ & $0.371^{\mathrm{g}}$ \\
\hline Coronary artery disease, $n(\%)$ & $3(8.8)$ & $3(11.1)$ & $0.766^{\mathrm{g}}$ \\
\hline ICAS, $n(\%)$ & $13(38.2)$ & $11(40.7)$ & $0.842^{\mathrm{f}}$ \\
\hline Stent placement, $n(\%)^{\mathrm{d}}$ & $3(23.1)$ & $1(9.1)$ & $0.596^{\mathrm{g}}$ \\
\hline Treated with tirofiban during EVT, $n(\%)^{\mathrm{d}}$ & $11(84.6)$ & $8(72.7)$ & $0.630^{\mathrm{g}}$ \\
\hline Dual antiplatelet therapy after EVT, $n(\%)^{\mathrm{d}}$ & $4(30.8)$ & $2(18.2)$ & $0.649^{\mathrm{g}}$ \\
\hline Overall early complications, $n(\%)$ & $21(61.8)$ & $0(0)$ & $<0.001^{\mathrm{f}}$ \\
\hline $\mathrm{END}, n(\%)$ & $20(58.8)$ & $0(0)$ & $<0.001^{\mathrm{f}}$ \\
\hline MBE, $n(\%)$ & $15(44.1)$ & $0(0)$ & $<0.001^{\mathrm{f}}$ \\
\hline Brain herniation, $n(\%)$ & $15(44.1)$ & $0(0)$ & $<0.001^{\mathrm{f}}$ \\
\hline $\mathrm{sHT}, n(\%)$ & $9(26.5)$ & $0(0)$ & $0.003^{\mathrm{g}}$ \\
\hline $\mathrm{HT}, n(\%)$ & $13(38.2)$ & $7(25.9)$ & $0.309^{\mathrm{f}}$ \\
\hline
\end{tabular}

AIS, acute ischemic stroke; EVT, endovascular thrombectomy; IQR, interquartile range; NIHSS, National Institute of Health Stroke Scale; ASPECTS, Alberta Stroke Program Early CT Score; SBP, systolic blood pressure; DBP, diastolic blood pressure; tICA, terminal internal carotid artery; MCA, middle cerebral artery; TOAST, Trial of Org 10,172 in Acute Stroke Treatment; ICAS, intracranial atherosclerotic stenosis; END, early neurological deterioration; sHT, symptomatic hemorrhagic transformation; MBE, malignant brain edema; SD, standard deviation. ${ }^{\text {a }}$ The mean value of SBP, measured at regular 60 -min intervals within the first $24 \mathrm{~h}$ after EVT. ${ }^{\mathrm{b}}$ The mean value of DBP, measured at regular 60 -min intervals within the first $24 \mathrm{~h}$ after EVT. ${ }^{\mathrm{c}} \mathrm{SBP}, \max \geq 160 \mathrm{~mm}$ $\mathrm{Hg}$ within the first $24 \mathrm{~h}$ after EVT. ${ }^{\mathrm{d}}$ Among patients with ICAS. ${ }^{\mathrm{e}}$ Mann-Whitney U tests for unpaired groups. ${ }^{\mathrm{f}}$ Pearson's $\chi^{2}$ tests. ${ }^{\mathrm{g}}$ Fisher's exact tests. ${ }^{\text {h }}$ Student's $t$ tests. 
Table 2. Clinical characteristics of AIS patients who achieved successful recanalization by EVT between those with and without overall early complications

\begin{tabular}{|c|c|c|c|}
\hline \multirow[t]{2}{*}{ Clinical characteristics } & \multicolumn{2}{|c|}{ Overall early complications } & \multirow[t]{2}{*}{$p$ value } \\
\hline & yes $(n=19)$ & no $(n=40)$ & \\
\hline Age, median (IQR), years & $62(52-80)$ & $51(40-62)$ & $0.005^{\mathrm{e}}$ \\
\hline Initial NIHSS score, median (IQR) & $18(14-20)$ & $14(12-18)$ & $0.017^{\mathrm{e}}$ \\
\hline Time from onset to recanalization, median (IQR), min & $324(295-438)$ & $378(250-485)$ & $0.615^{\mathrm{e}}$ \\
\hline Admission ASPECTS, median (IQR) & $8(6-8)$ & $8(7-9)$ & $0.137^{\mathrm{e}}$ \\
\hline Male, $n(\%)$ & $13(68.4)$ & $28(70.0)$ & $0.902^{\mathrm{f}}$ \\
\hline Treatment with intravenous thrombolysis, $n(\%)$ & $9(47.4)$ & $14(35.0)$ & $0.363^{\mathrm{f}}$ \\
\hline Number of passes $\geq 4$ times, $n(\%)$ & $4(21.1)$ & $2(5.0)$ & $0.078^{\mathrm{g}}$ \\
\hline Mean SBP, mean (SD), mm Hg ${ }^{\mathrm{a}}$ & $131(8)$ & $127(14)$ & $0.199^{\mathrm{h}}$ \\
\hline Mean DBP, mean (SD), mm Hg & $73(9)$ & $73(10)$ & $0.982^{\mathrm{h}}$ \\
\hline $\mathrm{SBP} \max \geq 160 \mathrm{~mm} \mathrm{Hg}, n(\%)^{\mathrm{c}}$ & $9(47.4)$ & $12(30.0)$ & $0.193^{\mathrm{f}}$ \\
\hline Fasting blood glucose, $\mathrm{mmol} / \mathrm{L}$ & $7.5(6.4-8.8)$ & $6.7(5.8-8.2)$ & $0.250^{\mathrm{e}}$ \\
\hline Site of occlusion & - & - & $0.639^{9}$ \\
\hline tICA, $n(\%)$ & $5(26.3)$ & $6(15.0)$ & - \\
\hline MCA-M1 segment, $n(\%)$ & $13(68.4)$ & $30(75.0)$ & - \\
\hline MCA-M2 segment, $n(\%)$ & $1(5.3)$ & $4(10.0)$ & - \\
\hline Stroke subtype by TOAST criteria & - & - & $0.708^{\mathrm{g}}$ \\
\hline Large-artery atherosclerosis, $n(\%)$ & $6(31.6)$ & $18(45.0)$ & - \\
\hline Cardioembolism, $n(\%)$ & $10(52.6)$ & $14(35.0)$ & - \\
\hline Stroke of other determined etiology, $n(\%)$ & $1(5.3)$ & $3(7.5)$ & - \\
\hline Stroke of undetermined etiology, $n(\%)$ & $2(10.5)$ & $5(12.5)$ & - \\
\hline Hypertension, $n(\%)$ & $10(52.6)$ & $16(40.0)$ & $0.361^{\mathrm{f}}$ \\
\hline Diabetes mellitus, $n(\%)$ & $3(15.8)$ & $8(20.0)$ & $0.976^{\mathrm{g}}$ \\
\hline Hyperlipemia, $n(\%)$ & $2(10.5)$ & $9(22.5)$ & $0.456^{\mathrm{g}}$ \\
\hline Atrial fibrillation, $n(\%)$ & $6(31.6)$ & $10(25.0)$ & $0.595^{\mathrm{g}}$ \\
\hline Smoke, $n(\%)$ & $6(31.6)$ & $15(37.5)$ & $0.657^{\mathrm{f}}$ \\
\hline Congestive heart failure, $n(\%)$ & $3(15.8)$ & $2(5.0)$ & $0.316^{\mathrm{g}}$ \\
\hline Coronary artery disease, $n(\%)$ & $3(15.8)$ & $3(7.5)$ & $0.376^{\mathrm{g}}$ \\
\hline ICAS, $n(\%)$ & $6(31.6)$ & $18(45.0)$ & $0.327^{\mathrm{f}}$ \\
\hline Stent placement, $n(\%)^{\mathrm{d}}$ & $0(0.0)$ & $4(22.2)$ & $0.539^{\mathrm{g}}$ \\
\hline Treated with tirofiban during EVT, $n(\%)^{\mathrm{d}}$ & $6(100.0)$ & $13(72.2)$ & $0.280^{\mathrm{g}}$ \\
\hline Dual antiplatelet therapy after EVT, $n(\%)^{\mathrm{d}}$ & $0(0.0)$ & $6(33.3)$ & $0.277^{\mathrm{g}}$ \\
\hline
\end{tabular}

AIS, acute ischemic stroke; EVT, endovascular thrombectomy; IQR, interquartile range; NIHSS, National Institute of Health Stroke Scale; ASPECTS, Alberta Stroke Program Early CT, Score; SBP, systolic blood pressure; DBP, diastolic blood pressure; tICA, terminal internal carotid artery; MCA, middle cerebral artery; TOAST, Trial of Org 10,172 in Acute Stroke Treatment; ICAS, intracranial atherosclerotic stenosis; SD, standard deviation. a The mean value of SBP, measured at regular 60-min intervals within the first $24 \mathrm{~h}$ after EVT. ${ }^{\mathrm{b}}$ The mean value of DBP, measured at regular 60-min intervals within the first $24 \mathrm{~h}$ after EVT. ${ }^{\mathrm{c}}$ Maximal SBP, value $\geq 160 \mathrm{~mm} \mathrm{Hg}$ within the first $24 \mathrm{~h}$ after EVT. ${ }^{\mathrm{d}}$ Among patients with ICAS. ${ }^{\mathrm{e}}$ Mann-Whitney U tests for unpaired groups. ${ }^{\mathrm{f}}$ Pearson's $\chi^{2}$ tests. ${ }^{\mathrm{g}}$ Fisher's exact tests. ${ }^{\mathrm{h}}$ Student's $t$ tests.

time from recanalization to the occurrence of END, sHT, brain herniation and MBE are 39 (IQR 29-57) h, 32 (IQR 26-41) h, 36 (IQR 27-51) h and 37 (IQR 27-50) h, respectively. We found that the rates of END, MBE, brain herniation, or sHT were significantly higher in patients with futile recanalization than those without $(p<0.05)$ (Table 1).

Outcome Biomarkers in AIS Patients after EVT
Tables 1 and 2 describe the clinical characteristics of the patients between those with and without unfavorable outcomes (futile recanalization and overall early complications, respectively). Patients with futile recanalization or overall early complications were older and had higher initial NIHSS significantly than those without (all $p<$ $0.05)$. No significant differences between other characteristics and unfavorable outcomes were found (Tables 1,2). 
Table 3. Univariate and multivariate associations of plasma biomarkers with unfavorable outcomes in AIS patients who achieved successful recanalization by EVT

\begin{tabular}{lllll}
\hline & OR $(95 \% \mathrm{CI})$ & $p$ value & Adjusted OR (95\% CI) & $p$ value \\
\hline Overall early complications $(n=59)$ & & & & \\
$\quad$ MMP-9 & $2.416(1.460-3.997)$ & 0.001 & $2.464(1.383-4.388)$ & 0.002 \\
Tenascin-C & $4.539(1.605-12.837)$ & 0.004 & $3.443(1.176-10.077)$ & 0.024 \\
CRP & $2.318(1.339-4.013)$ & 0.003 & $1.918(1.063-3.462)$ & 0.031 \\
Thioredoxin & $5.125(1.522-17.253)$ & 0.008 & $3.314(1.054-10.425)$ & 0.040 \\
ADAMTS13 & $0.023(0.002-0.310)$ & 0.005 & $0.035(0.002-0.545)$ & 0.017 \\
Gelsolin & $0.040(0.005-0.303)$ & 0.002 & $0.060(0.006-0.640)$ & 0.020 \\
Futile recanalization $(n=61)$ & & & & \\
MMP-9 & $2.394(1.472-3.896)$ & $<0.001$ & $2.285(1.301-4.014)$ & 0.004 \\
Tenascin-C & $3.529(1.455-8.561)$ & 0.005 & $2.744(1.180-6.384)$ & 0.019 \\
CRP & $2.332(1.415-3.843)$ & 0.001 & $1.728(0.998-2.993)$ & 0.051 \\
Thioredoxin & $3.674(1.550-8.707)$ & 0.003 & $2.972(1.292-6.839)$ & 0.010 \\
ADAMTS13 & $0.031(0.002-0.390)$ & 0.007 & $0.042(0.002-0.794)$ & 0.035 \\
Gelsolin & $0.021(0.002-0.193)$ & 0.001 & $0.068(0.005-0.932)$ & 0.044 \\
\hline
\end{tabular}

AIS, acute ischemic stroke; CFP, C-reactive protein; EVT, endovascular thrombectomy; OR, odds ratio; CI, confidence interval; NIHSS, National Institute of Health Stroke Scale; MMP-9, matrix metalloproteinase-9. a Adjustment for age and initial NIHSS, score. The levels of all the biomarkers were recorded as continuous variable.

The rates of patients with intracranial atherosclerotic stenosis (ICAS) were comparable between those with and without futile recanalization (Pearson's $\chi^{2}$ test, $p=0.842$ ). Likewise, the rates of patients with ICAS were comparable between those with and without overall early complications (Pearson's $\chi^{2}$ test, $p=0.327$ ). According to transcranial Doppler sonography performed at 1 or 2 days after EVT, none of the patients included in this study were found to have experienced reocclusion after endovascular recanalization.

\section{Univariate and Multivariate Analysis}

Univariate analysis indicated that age and initial NIHSS were significantly associated with futile recanalization (age: OR 1.052, 95\% confidence interval [CI] 1.018$1.088, p=0.003$; NIHSS: OR 1.269, 95\% CI 1.095-1.470, $p=0.002$ ) and overall early complications (age: OR 1.045, 95\% CI 1.011-1.081, $p=0.009$; NIHSS: OR 1.147, 95\% CI $1.006-1.308, p=0.040)$. Multivariate analysis showed that age (aOR 1.041, 95\% CI 1.001-1.083, $p=0.046$ ) and initial NIHSS (aOR 1.284, 95\% CI 1.048-1.574, $p=0.016$ ) remained significantly associated with futile recanalization after adjusting for admission ASPECTS, mean SBP within $24 \mathrm{~h}$ after EVT, fasting blood glucose, atrial fibrillation, and number of passes. Furthermore, multivariate analysis showed that age (aOR 1.048, 95\% CI 1.004-1.093, $p=$ 0.031 ) and initial NIHSS (aOR 1.210, 95\% CI 1.001-1.461, $p=0.048)$ remained significantly associated with overall early complications after adjusting for admission ASPECTS, mean SBP within $24 \mathrm{~h}$ after EVT, fasting blood glucose, atrial fibrillation, and number of passes.

Univariate analysis of plasma biomarkers indicated that MMP-9, tenascin-C, CRP, thioredoxin, ADAMTS13, and gelsolin were significantly associated with both futile recanalization and overall early complications (all $p<$ 0.05 ) (Table 3). Multivariate analysis confirmed that increased levels of MMP-9, tenascin-C, CRP, and thioredoxin and decreased levels of ADAMTS13 and gelsolin remained significantly associated with futile recanalization and overall early complications after adjustment for age and initial NIHSS, except that CRP slightly missed the margin of significance in the association with futile recanalization (adjusted OR 1.728, 95\% CI 0.998-2.993, $p=$ 0.051) (Table 3).

\section{Discrimination and Reclassification}

We constructed BCM including age and initial NIHSS for unfavorable outcome discrimination. BCM revealed moderate discriminating ability for futile recanalization (AUC 0.807, 95\% CI 0.693-0.921) and weak discrimination for overall early complications (AUC $0.749,95 \%$ CI 0.611-0.887) (Table 4; Fig. 2). The AUC value of each biomarker is listed in online suppl. Table 2. None of single plasma biomarker addition significantly improved the 
Table 4. ROC curve analysis of the addition of plasma biomarkers to the BCM for prediction of unfavorable outcomes in AIS patients who achieved successful recanalization by EVT

\begin{tabular}{|c|c|c|c|c|c|c|}
\hline & \multicolumn{4}{|l|}{ Discrimination } & \multicolumn{2}{|l|}{ Reclassification } \\
\hline $\mathrm{BCM}^{\mathrm{a}}$ & $0.749(0.611-0.887)$ & - & - & - & - & - \\
\hline BCM+MMP-9+Tenascin-C & $0.868(0.766-0.970)$ & 0.039 & $19.7(7.9-31.6)$ & 0.001 & $59.1(23.8-94.4)$ & 0.001 \\
\hline BCM+MMP-9+CRP & $0.882(0.773-0.990)$ & 0.040 & $25.7(12.0-39.3)$ & $<0.001$ & $71.8(37.1-106.6)$ & $<0.001$ \\
\hline $\mathrm{BCM}+\mathrm{CRP}+\mathrm{ADAMTS} 13$ & $0.863(0.764-0.962)$ & 0.034 & $18.7(5.4-31.9)$ & 0.006 & $38.3(2.7-73.9)$ & 0.035 \\
\hline \multicolumn{7}{|l|}{ Futile recanalization $(n=61)$} \\
\hline $\mathrm{BCM}^{\mathrm{a}}$ & $0.807(0.693-0.921)$ & - & - & - & - & - \\
\hline BCM+MMP-9+Thioredoxin & $0.908(0.839-0.978)$ & 0.043 & $20.9(10.4-31.3)$ & $<0.001$ & $67.2(32.4-102.0)$ & $<0.001$ \\
\hline
\end{tabular}

AIS, acute ischemic stroke; EVT, endovascular thrombectomy; AUC, area under the curve; CI, confidence interval; ROC, receiver operating characteristic; NRI, net reclassification improvement; IDI, integrated discrimination improvement; MMP-9, matrix metalloproteinase-9; CRP, C-reactive protein. ${ }^{a}$ BCM, indicates baseline clinical model. ${ }^{b} p$ value for AUC, using the DeLong method with BCM, as a reference. ${ }^{c} p$ value for IDI with the BCM, as a reference. ${ }^{\mathrm{d}} p$ value for NRI with the BCM, as a reference.

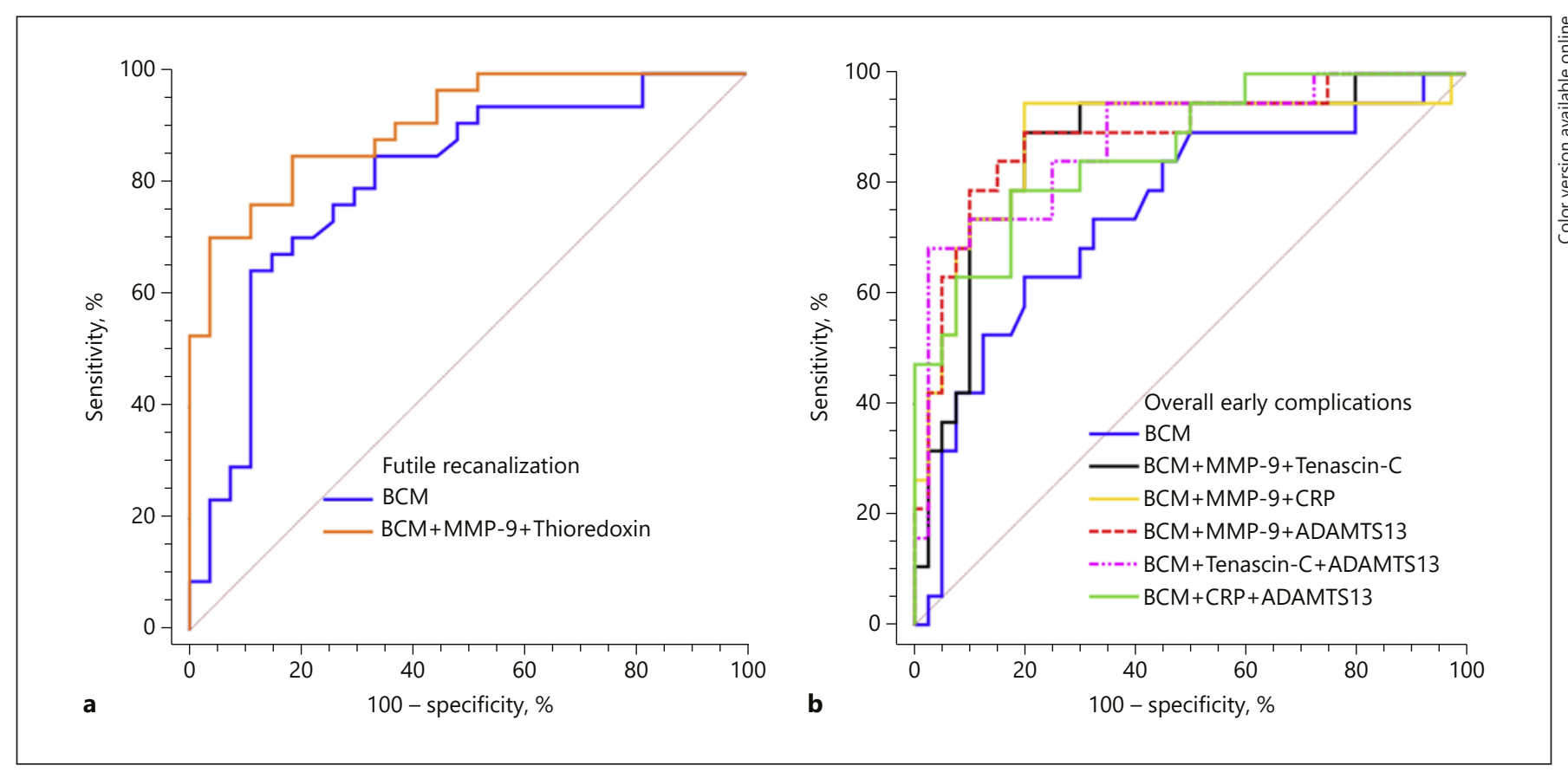

Fig. 2. ROC curves of the addition of the combined plasma biomarkers to the clinical baseline model for discriminating (a) futile recanalization and (b) overall early complications. BCM, baseline clinical model; MMP-9, matrix metalloproteinase-9; CRP, C-reactive protein; ROC, receiver operating characteristic.

AUC value of BCM to discriminate futile recanalization or overall early complications assessed by the DeLong test (online suppl. Table 3).

The panel of BCM+MMP-9+thioredoxin significantly enhanced the discrimination and reclassification of BCM for futile recanalization (AUC 0.908, $p=0.043$; IDI 20.9\%, $p<0.001$; NRI $67.2 \%, p<0.001)$. There were no multicollinearity problems between the tested variables in this model (Table 4). Five plasma biomarker panels in addition to BCM significantly improved the discrimination 
and reclassification of $\mathrm{BCM}$ for overall early complications: (1) BCM+MMP-9+tenascin-C (AUC 0.868, $p=$ 0.039; IDI 19.7\%, $p=0.001$; NRI 59.1\%, $p=0.001)$; (2) BCM+MMP-9+CRP (AUC 0.882, $p=0.040$; IDI 25.7\%, $p<0.001$; NRI 71.8\%, $p<0.001$ ); (3) BCM+MMP-9+ ADAMTS13 (AUC 0.886, $p=0.042$; IDI 23.4\%, $p=0.001$; NRI 51.1\%, $p=0.006) ;(4) \mathrm{BCM}+$ tenascin-C+ADAMTS13 (AUC 0.880, $p=0.036$; IDI 19.3\%, $p<0.001$; NRI 67.4\%, $p<0.001$ ); and (5) BCM+CRP+ADAMTS13 (AUC 0.863, $p=0.034$; IDI $18.7 \%, p=0.006$; NRI $38.3 \%, p=0.035)$. No multi-collinearity was found among the tested variables in the models above (Table 4; Fig. 2). Other combinations of biomarkers without multi-collinearity did not improve the discrimination of $\mathrm{BCM}$ for unfavorable outcomes tested by the DeLong method, and the results are shown in online suppl. Table 3.

\section{Discussion}

The present study investigated the utility of several biomarker panels to predict early complications and futile recanalization following EVT. Our findings suggest that changes in specific plasma biomarkers after EVT improve the discriminating ability to predict unfavorable outcome and futile recanalization at 90 days.

$\mathrm{BBB}$ breakdown is mediated by the release of various proteases, particularly matrix metalloproteases (MMPs) [31]. MMP-9, a member of MMPs family, is a well-established marker of BBB disruption. Increased level of MMP9 is related to vasogenic edema formation, $\mathrm{sHT}$, and functional dependence after 3 months for AIS patients [18, 19].

Tenascin-C, a glycoprotein expressed in extracellular matrix, is involved in maintaining the basement membranes of BBB [32, 33]. Experimental research found that tenascin- $C$ was implicated in neuronal apoptosis and $\mathrm{BBB}$ disruption via TLR4/NF- $\kappa \mathrm{B} / \mathrm{IL}-1 \beta$ inhibition in cerebrovascular diseases $[21,34]$. Recent clinical studies demonstrated that enhanced serum tenascin-C level was associated with poor outcomes of patients with acute hemorrhage stroke [35].

CRP is a well-acknowledged inflammatory biomarker, playing a key role in ischemia-reperfusion injury in AIS. The circulating level of CRP has been proved to be associated with functional outcome at 3 months in AIS patients $[22,23]$. Moreover, CRP reflects not only inflammation triggered by cerebral ischemia but also post-stroke infection or other inflammatory stimuli, especially in critically ill patients.
Thioredoxin is a good indicator of oxidative stress in ischemia-reperfusion injury in brain, heart and endothelium [24-26]. Furthermore, inhibition of thioredoxin was found to be involved in attenuating BBB disruption in the early stage of experimental focal cerebral ischemia [36]. Recent clinical studies showed that elevated serum thioredoxin level on admission can predict functional independence at 3 months after AIS [37-39].

ADAMTS13 is a metalloprotease enzyme, cleaving ultra-large von Willebrand factor multimers into smaller, less active forms [27]. Decreased level of ADAMTS13 was associated with poor functional outcomes for AIS patients $[7,40]$. The neuroprotective effect of ADAMTS13 may be explained by its antithrombotic role in microcirculation. After recanalization of the large vessel, the reperfusion of penumbra area may remain poor due to microthrombosis or microemboli as a result of platelet adhesion and leukocyte recruitment mediated by ultra-large von Willebrand factor multimers, which cannot be cleaved sufficiently with a low level of ADAMTS13 [41, 42].

Gelsolin is an actin-depolymerizing protein that scavenges actin leaking into the bloodstream after tissue injury [11]. Convincing evidence indicates that decreased gelsolin on admission was an independent predictor of poor outcomes in patients with AIS and intracerebral hemorrhage $[43,44]$. In response to ischemia injury, high amounts of filamentous actin (F-actin) was released from damaged cells into bloodstream, leading to the increase of blood viscosity, disturbances in microvascular flow, and impairment of fibrinolysis [28]. Gelsolin may be crucial to limit microvascular thrombosis via depolymerization of F-actin in bloodstream after recanalization in AIS.

To date, there are scarce studies regarding the predictive value of plasma biomarkers mentioned above in AIS patients after EVT. Our results showed that increased levels of MMP-9, tenascin-C, CRP, and thioredoxin and decreased levels of ADAMTS13 and gelsolin were independent predictors of futile recanalization after adjustment for clinical variables in AIS patients who achieved recanalization via EVT.

Our study investigated for the first time the importance of several plasma biomarkers associated with early complications and futile recanalization in patients with successful recanalization by EVT. However, some limitations should be noticed. The first limitation resides in the relatively small number of patients due to strict inclusion criteria, for instance, patients with unsuccessful vessel recanalization after thrombectomy (mTICI $<2$ b) were not included in this study, the intention of which was to as- 
sure homogeneity among the patients enrolled. Second, there may be an underlying overlap of blood collection and the initiation of complications because we could not rule out the possibility that an early complication had already initiated without classical clinical manifestation at the moment of sampling for some patients. Thus, we cannot draw a conclusion that plasma biomarkers measured at $18-24 \mathrm{~h}$ after EVT were predictors of early complications. Third, the plasma biomarkers for measurements were performed at 1 single time point, without investigating the dynamic evolution after EVT. Serial measurements could give the opportunity to explore whether persistent higher levels of biomarkers remained predictive for outcomes, which can be explored in further research.

\section{Conclusions}

For anterior LVO AIS patients with recanalization by EVT, the increased levels of MMP-9, tenascin-C, CRP, and thioredoxin and decreased levels of ADAMTS13 and gelsolin were independent predictors of futile recanalization. The panel of BCM+MMP-9+thioredoxin can improve the discrimination of futile recanalization, and the panels of BCM+MMP-9+tenascin-C, BCM+MMP-9+ CRP, BCM+MMP-9+ADAMTS13, BCM+tenascin-C+ ADAMTS13, and BCM+CRP+ADAMTS13 all have additive clinical value for discriminating overall early complications. Incorporation of biomarker panels can substantially improve the risk stratification for poor AIS outcomes after recanalization by EVT.

\section{Statement of Ethics}

All the authors state that all the subjects (or their guardians) have given their written informed consent and that the study protocol was approved by the Medical Ethics Committee of NanFang Hospital (NFEC-2019-189).

\section{Conflict of Interest Statement}

None of the authors have conflict of interest to declare.

\section{Funding Sources}

This study was supported by the National Key R\&D Program of China (No. 2017YFC1307500), National Natural Science Foundation of China (No. 81701294 \& 81871030), Natural Science Foundation of Guangdong Province (No. 2019A1515011446), Guangzhou Science and Technology Planning Project (No. 201804010055), Guangdong Province Aid of Xinjiang Rural Science and Technology Development (Special Commissioner) Project (KTP20190278), and Pilot Project of Technology Promotion and Poverty Alleviation by National Health Commission (2019JSTG31).

\section{Author Contributions}

N.L.Z. and Z.Z.L.: data analysis and manuscript writing; K.B.H. and Y.P.: patient recruitment and manuscript revision; Y.N.W., Y.M.W., S.N.W., and D.M.W.: data acquisition; S.Y.P. and Z.J.: conception, study design, and manuscript revision.

\section{References}

1 Saver JL, Goyal M, van der Lugt A, Menon BK, Majoie CB, Dippel DW, et al. Time to treatment with endovascular thrombectomy and outcomes from ischemic stroke: a meta-analysis. JAMA. 2016 Sep;316(12):1279-88.

2 Kim JM, Bae JH, Park KY, Lee WJ, Byun JS, Ahn SW, et al. Incidence and mechanism of early neurological deterioration after endovascular thrombectomy. J Neurol. 2019 Mar; 266(3):609-15

3 Hao Y, Yang D, Wang H, Zi W, Zhang M, Geng Y, et al. Predictors for symptomatic intracranial hemorrhage after endovascular treatment of acute ischemic stroke. Stroke. 2017 May;48(5):1203-9.

4 Beez Thomas, Munoz-Bendix Christopher, Steiger Hans-Jakob, Beseoglu Kerim. Decompressive craniectomy for acute ischemic stroke. Crit Care. 2019;23(1):209.
5 Huang X, Yang Q, Shi X, Xu X, Ge L, Ding X, et al. Predictors of malignant brain edema after mechanical thrombectomy for acute ischemic stroke. J Neurointerv Surg. 2019 Oct; 11(10):994-8

6 Nie X, Pu Y, Zhang Z, Liu X, Duan W, Liu L. Futile recanalization after endovascular therapy in acute ischemic stroke. Biomed Res Int. 2018 May;2018:5879548.

7 Bustamante A, Ning M, García-Berrocoso T, Penalba A, Boada C, Simats A, et al. Usefulness of ADAMTS13 to predict response to recanalization therapies in acute ischemic stroke. Neurology. 2018 Mar;90(12):e9951004.

8 Gilberti N, Gamba M, Premi E, Costa A, Vergani V, Delrio I, et al. Leukoaraiosis is a predictor of futile recanalization in acute ischemic stroke. J Neurol. 2017 Mar;264(3):44852.
9 Hussein HM, Georgiadis AL, Vazquez G, Miley JT, Memon MZ, Mohammad YM, et al. Occurrence and predictors of futile recanalization following endovascular treatment among patients with acute ischemic stroke: a multicenter study. AJNR Am J Neuroradiol. 2010 Mar;31(3):454-8

10 Park SY, Kim J, Kim OJ, Kim JK, Song J, Shin $\mathrm{DA}$, et al. Predictive value of circulating interleukin-6 and heart-type fatty acid binding protein for three months clinical outcome in acute cerebral infarction: multiple blood markers profiling study. Crit Care. 2013 Mar; 17(2):R45.

11 Garcia-Berrocoso T, Penalba A, Boada C, Giralt $\mathrm{D}$, Cuadrado E, Colome N, et al. From brain to blood: new biomarkers for ischemic stroke prognosis. J Proteomics. 2013 Dec;94: $138-48$. 
12 Yoo AJ, Simonsen CZ, Prabhakaran S, Chaudhry ZA, Issa MA, Fugate JE, et al. Refining angiographic biomarkers of revascularization: improving outcome prediction after intra-arterial therapy. Stroke. 2013 Sep; 44(9):2509-12.

13 Jauch EC, Saver JL, Adams HP Jr, Bruno A, Connors JJ, Demaerschalk BM, et al. Guidelines for the early management of patients with acute ischemic stroke: a guideline for healthcare professionals from the American Heart Association/American Stroke Association. Stroke. 2013 Mar;44(3):870-947.

14 Albers GW, Marks MP, Kemp S, Christensen S, Tsai JP, Ortega-Gutierrez S, et al. DEFUSE 3 investigators. thrombectomy for stroke at 6 to 16 hours with selection by perfusion imaging. N Engl J Med. 2018 Feb;378(8):708-18.

15 Simonsen CZ, Schmitz ML, Madsen MH, Mikkelsen IK, Chandra RV, Leslie-Mazwi T, et al. Early neurological deterioration after thrombolysis: clinical and imaging predictors. Int J Stroke. 2016 Oct;11(7):776-82.

16 Wu C, Sun C, Wang L, Lian Y, Xie N, Huang $S$, et al. Low-dose tirofiban treatment improves neurological deterioration outcome after intravenous thrombolysis. Stroke. 2019 Dec;50(12):3481-7.

17 Xing B, Ma J, Jiang Z, Feng Z, Ling S, Szigety $\mathrm{K}$, et al. GLP-1 signaling suppresses menin's transcriptional block by phosphorylation in $\beta$ cells. J Cell Biol. 2019 Mar;218(3):855-70.

18 Ramos-Fernandez M, Bellolio MF, Stead LG. Matrix metalloproteinase- 9 as a marker for acute ischemic stroke: a systematic review. J Stroke Cerebrovasc Dis. 2011 Jan;20(1):4754.

19 Barr TL, Latour LL, Lee KY, Schaewe TJ, Luby M, Chang GS, et al. Blood-brain barrier disruption in humans is independently associated with increased matrix metalloproteinase-9. Stroke. 2010 Mar;41(3):e123-8.

20 Suzuki H, Fujimoto M, Kawakita F, Liu L, Nakatsuka Y, Nakano F, et al. Tenascin-C in brain injuries and edema after subarachnoid hemorrhage: findings from basic and clinical studies. J Neurosci Res. 2020 Jan;98(1):42-56.

21 Fujimoto M, Shiba M, Kawakita F, Liu L, Shimojo N, Imanaka-Yoshida K, et al. Deficiency of tenascin-C and attenuation of blood-brain barrier disruption following experimental subarachnoid hemorrhage in mice. J Neurosurg. 2016 Jun;124(6):1693-702.

22 Huțanu A, Iancu M, Bălaşa R, Maier S, Dobreanu M. Predicting functional outcome of ischemic stroke patients in Romania based on plasma CRP, sTNFR-1, D-dimers, NGAL and NSE measured using a biochip array. Acta Pharmacol Sin. 2018 Jul;39(7):1228-36.
23 Seo WK, Seok HY, Kim JH, Park MH, Yu SW, $\mathrm{Oh} \mathrm{K}$, et al. C-reactive protein is a predictor of early neurologic deterioration in acute ischemic stroke. J Stroke Cerebrovasc Dis. 2012 Apr;21(3):181-6.

24 Li L, Zhu K, Liu Y, Wu X, Wu J, Zhao Y, et al. Targeting thioredoxin-1 with siRNA exacerbates oxidative stress injury after cerebral ischemia/reperfusion in rats. Neuroscience. 2015 Jan;284:815-23.

25 Liu Y, Ma Y, Wang R, Xia C, Zhang R, Lian K, et al. Advanced glycation end products accelerate ischemia/reperfusion injury through receptor of advanced end product/nitrative thioredoxin inactivation in cardiac microvascular endothelial cells. Antioxid Redox Signal. 2011 Oct;15(7):1769-78.

26 Wang BF, Yoshioka J. The emerging role of thioredoxin-interacting protein in myocardial ischemia/reperfusion injury. J Cardiovasc Pharmacol Ther. 2017 May;22(3):21929.

27 Xu H, Cao Y, Yang X, Cai P, Kang L, Zhu X, et al. ADAMTS13 controls vascular remodeling by modifying VWF reactivity during stroke recovery. Blood. 2017 Jul;130(1):1122.

28 Lee WM, Galbraith RM. The extracellular actin-scavenger system and actin toxicity. $\mathrm{N}$ Engl J Med. 1992 May;326(20):1335-41.

29 Piktel E, Levental I, Durnaś B, Janmey PA, Bucki R. Plasma gelsolin: indicator of inflammation and its potential as a diagnostic tool and therapeutic target. Int J Mol Sci. 2018 Aug;19(9):2516.

30 Di Castelnuovo A, Veronesi G, Costanzo S, Zeller T, Schnabel RB, de Curtis A, et al. NTproBNP ( $\mathrm{N}$-terminal pro-B-type natriuretic peptide) and the risk of stroke. Stroke. 2019 Mar;50(3):610-7.

31 Sifat AE, Vaidya B, Abbruscato TJ. Bloodbrain barrier protection as a therapeutic strategy for acute ischemic stroke. AAPS J. 2017 Jul;19(4):957-72.

32 Baeten KM, Akassoglou K. Extracellular matrix and matrix receptors in blood-brain barrier formation and stroke. Dev Neurobiol. 2011 Nov;71(11):1018-39.

33 Garwood J, Garcion E, Dobbertin A, Heck N, Calco V, ffrench-Constant C, et al. The extracellular matrix glycoprotein Tenascin-C is expressed by oligodendrocyte precursor cells and required for the regulation of maturation rate, survival and responsiveness to plateletderived growth factor. Eur J Neurosci. 2004 Nov;20(10):2524-40.
34 Liu L, Fujimoto M, Nakano F, Nishikawa H, Okada T, Kawakita F, et al. Deficiency of tenascin-C alleviates neuronal apoptosis and neuroinflammation after experimental subarachnoid hemorrhage in mice. Mol Neurobiol. 2018 Nov;55(11):8346-54

35 Wang LG, Huangfu XQ, Tao B, Zhong GJ, Le $Z D$. Serum tenascin-C predicts severity and outcome of acute intracerebral hemorrhage. Clin Chim Acta. 2018 Jun;481:69-74.

36 Chi OZ, Barsoum S, Grayson J, Liu X, Weiss HR. Effects of the thioredoxin-1 inhibitor PX12 on blood-brain barrier permeability in the early stage of focal cerebral ischemia. Pharmacology. 2013;92(3-4):175-81.

37 Qi AQ, Li Y, Liu Q, Si JZ, Tang XM, Zhang $Z Q$, et al. Thioredoxin is a novel diagnostic and prognostic marker in patients with ischemic stroke. Free Radic Biol Med. 2015 Mar; 80:129-35.

$38 \mathrm{Wu}$ MH, Song FY, Wei LP, Meng ZY, Zhang ZQ, Qi QD. Serum levels of thioredoxin are associated with stroke risk, severity, and lesion volumes. Mol Neurobiol. 2016 Jan;53(1): $677-85$.

39 Yu T, Zhang W, Lin Y, Li Q, Xue J, Cai Z, et al. Prognostic value of serum thioredoxin levels in ischemic stroke. Neurol Res. 2017 Nov; 39(11):988-95.

40 Prochazka V, Jonszta T, Czerny D, Krajca J, Roubec M, Macak J, et al. The role of von Willebrand factor, ADAMTS13, and cerebral artery thrombus composition in patient outcome following mechanical thrombectomy for acute ischemic stroke. Med Sci Monit. 2018 Jun;24:3929-45.

41 Fujioka M, Hayakawa K, Mishima K, Kunizawa A, Irie K, Higuchi S, et al. ADAMTS13 gene deletion aggravates ischemic brain damage: a possible neuroprotective role of ADAMTS13 by ameliorating postischemic hypoperfusion. Blood. 2010 Feb;115(8): 1650-3.

42 Kleinschnitz C, De Meyer SF, Schwarz T, Austinat M, Vanhoorelbeke K, Nieswandt B, et al. Deficiency of von Willebrand factor protects mice from ischemic stroke. Blood. 2009 Apr;113(15):3600-3.

43 Guo XC, Luo BY, Li XF, Yang DG, Zheng XN Zhang K. Plasma gelsolin levels and 1-year mortality after first-ever ischemic stroke. J Crit Care. 2011 Dec;26(6):608-12.

44 Zhao DQ, Wang K, Zhang HD, Li YJ. Significant reduction of plasma gelsolin levels in patients with intracerebral hemorrhage. Clin Chim Acta. 2013 Jan;415:202-6. 